\title{
Inmersividad analógica: descubriendo el dibujo esférico entre subjetividad y objetividad
}

\author{
Analogical immersion: discovering spherical sketches between \\ subjectivity and objectivity
}

\section{Resumen}

Autor:

Lucas Fabián Olivero *

lucasfabian.olivero@

unicampania.it

Bruno Sucurado**

brunosucurado@gmail.com

* Università della Campania Luigi Vanvitell

** Universidad Nacional de la Plata

* Italia

** Argentina

Recibido:4/Abr/2018 Aceptado: 24/Feb/2019
$\mathrm{E}$ I uso de la representación gráfica analógica se ha visto reducido frente a la potente representación digital. Sin embargo, los largos años de historia con los que cuenta generan nuevos caminos de evolución. Como resultado, lo autógrafo cala hondo en la complejidad digital y se funde con ella. Las producciones aquí detalladas, que se nutren de arquitectura, ingeniería e informática, oscilan entre la impresión subjetiva y la reinterpretación de conceptos base de la perspectiva. Tienen por objeto compartir y difundir aplicaciones, tanto profesionales como en las aulas. En lo específico, se plantea la búsqueda intuitiva y experimental: el pensamiento gráfico de una representación espacial inmersiva. Esta singular representación plasma en las dos dimensiones de un plano el despliegue característico de una superficie (en particular, la de la esfera y la del cubo) centrada en el observador. Es decir, trabaja con dos de las anamorfosis más frecuentes que la tecnología digital usa para contenidos de realidad virtual. El objetivo es abrir las puertas a nuevos instrumentos híbridos destinados al proyecto y al levantamiento arquitectónico, así como también al arte y las disciplinas gráficas en general.

Palabras clave: dibujo analógico, dibujo autógrafo, proyección cúbica, proyección equirectangular, inmersividad, realidad virtual.

\section{Abstract:}

The use of analog graphics has been reduced compared to powerful digital representation. However, the long history of the company generates new paths of evolution. As a result, the autograph goes deep into digital complexity and merges with it. The productions detailed here, which are nurtured by architecture, engineering and information technology, oscillate between subjective impression and the reinterpretation of basic concepts of perspective. They aim to share and disseminate applications, both professional and in the classroom. Specifically, the intuitive and experimental search is proposed: the graphic thought of an immersive spatial representation. This singular representation captures in the two dimensions of a plane the characteristic unfolding of a surface (in particular, that of the sphere and that of the cube) centered on the observer. In other words, it works with two of the most frequent anamorphoses that digital technology uses for virtual reality contents. The aim is to open the doors to new hybrid instruments for design and architectural uplifting, as well as for art and graphic disciplines in general.

Keywords: Analog drawing, autograph drawing, cubic projection, equirectangular projection, inmersion, virtual reality. 


\section{Introducción}

La historia de la representación gráfica ha evolucionado de la expresión pictórica rupestre en las cavernas al uso de la tecnología digital. Mientras que la primera resulta de composición, complejidad y significados más elementares, la segunda ha permitido una explosión de la expresividad y la complejidad compositiva. En particular, la caracterización de la expresión gráfica digital brinda la posibilidad casi íntegra de reversibilidad de su propio proceso creativo.

A mitad de ese camino, se desarrollarán las perspectivas a método y Piero Della Francesca iluminará el Renacimiento con su tratado De prospectiva pingendi (Della Francesca, 1474 aprox.), que cambiarán la forma de percibir el espacio y de hacer arte. Aparecerán también los primeros tratados sobre la anamorfosis, como el Perspectiva pictorum et architectorum (Pozzo, 1693). La teoría copernicana abrirá la mente a un radical nuevo concepto: el mundo es esférico y ya no plano. A tener en cuenta el problema de su representación será la cartografía la que vendrá a colación.

Entre el siglo XVIII y XIX se pasará a los primeros intentos de crear ambientes inmersivos con los panoramas pintados a 360 grados en las instalaciones de la rotonda panorámica de Robert Baker y con las proyecciones múltiples del Cinerama (Rossi, 2018; Cabezos Bernal, Cisneros Vivó y Soler Sanz, 2014). La inmersión es una actividad que tiene por objeto simular un entorno que no es real, para lo cual se crea un ambiente virtual que zpotencialmente puede existir y que "engaña" total o parcialmente al visitador

Ya en el siglo XX, hasta el mismo Dalí usará la estereoscopía y la anamorfosis para acentuar su representación surrealista.

En todo este camino, tanto la perspectiva como la estereoscopía fueron codificadas. No son exhaustivos, sin embargo, los tentativos de codificar la proyección esférica y menos aún los de aplicarla al estudio del espacio. Podemos citar aquí La perspective curviligne (Barre, Flocon y Bouligand, 1967) y los más recientes y avanzados estudios sobre la anamorfosis, la matemática y la representación espacial con uso de proyecciones esféricas como La prospettiva e la costruzione dello spazio figurativo (Masetti, 2014) acompañado de publicaciones como Drawing Equirectangular VR Panoramas y Constructing a total spherical perspective (Araújo, 2018a, 2018b).

El diseño gráfico, por su parte, desde nuestras actividades cotidianas, es interactivo en tiempo real. La idea de iconografía pasa a ser fundamental en las estrategias de desarrollo y presentación de proyectos, coronando con la idea del "compartir" en las redes, el eje de la idiosincrasia mundial contemporánea.

Gracias al uso del teléfono celular, crece exponencialmente la idea de estar "inmerso"; la visita virtual de lugares remotos queda al alcance de la mano gracias a la tecnología de Google Street View. Con ello se facilita también la elaboración de panoramas fotográficos gracias a la creación de apps específicas, germen de la representación con múltiples puntos de fuga.

Desde nuestra óptica, un profesional debería nutrirse equilibradamente de la complejidad y la simplicidad. No podemos, por lo tanto, dejar de alentar a que las próximas generaciones puedan ver la génesis, sea de los procesos analógicos o de los digitales, siendo la coyuntura de ambos el campo más libre de creatividad (y por ende de diversidad).

Las bases para una gran difusión de los dibujos inmersivos a 360 grados ya están dadas: por una parte, el conocimiento de base lo constituyen la perspectiva y la geometría descriptiva; por otra, Facebook, RoundMe y Wordpress (entre muchos otros). Gracias a que contienen instrumentos inicialmente codificados para fotos panorámicas, soportan sin inconvenientes dibujos panorámicos.

La forma de representar contemporánea se asocia inevitablemente a lo digital. Queremos, sin embargo, provocar una brecha proponiendo un camino híbrido basado en la experimentación con la anamorfosis, la tecnología digital y el dibujo autógrafo. Así, serán quizás estos caminos los que alienten a desarrollar tratados inmersivos. Lo único que necesitamos es mirar de manera diferente lo mismo que hemos visto hasta ahora.

\section{2. ¿Qué es un dibujo a $360^{\circ}$ ?}

Cuando hablamos de dibujos a 360 grados, hablamos de dibujos que no se concentran en representar solamente un fragmento, sino la integridad de lo que nos rodea. Para ser factible, nuestro tradicional cuadro de dibujo deja de ser un plano para pasar a ser una superficie tridimensional centrada en el observador. Es decir, que un dibujo a 360 grados es el dibujo plano (una anamorfosis 2D) de las caras internas de una superficie vista desde su interior.

La superficie elegida es clave para la integración con e mundo digital, donde la realidad virtual ha tenido su mayor crecimiento (environmental mapping). Dos de las superficies utilizadas son la esfera y el cubo (Greene, 1986). 


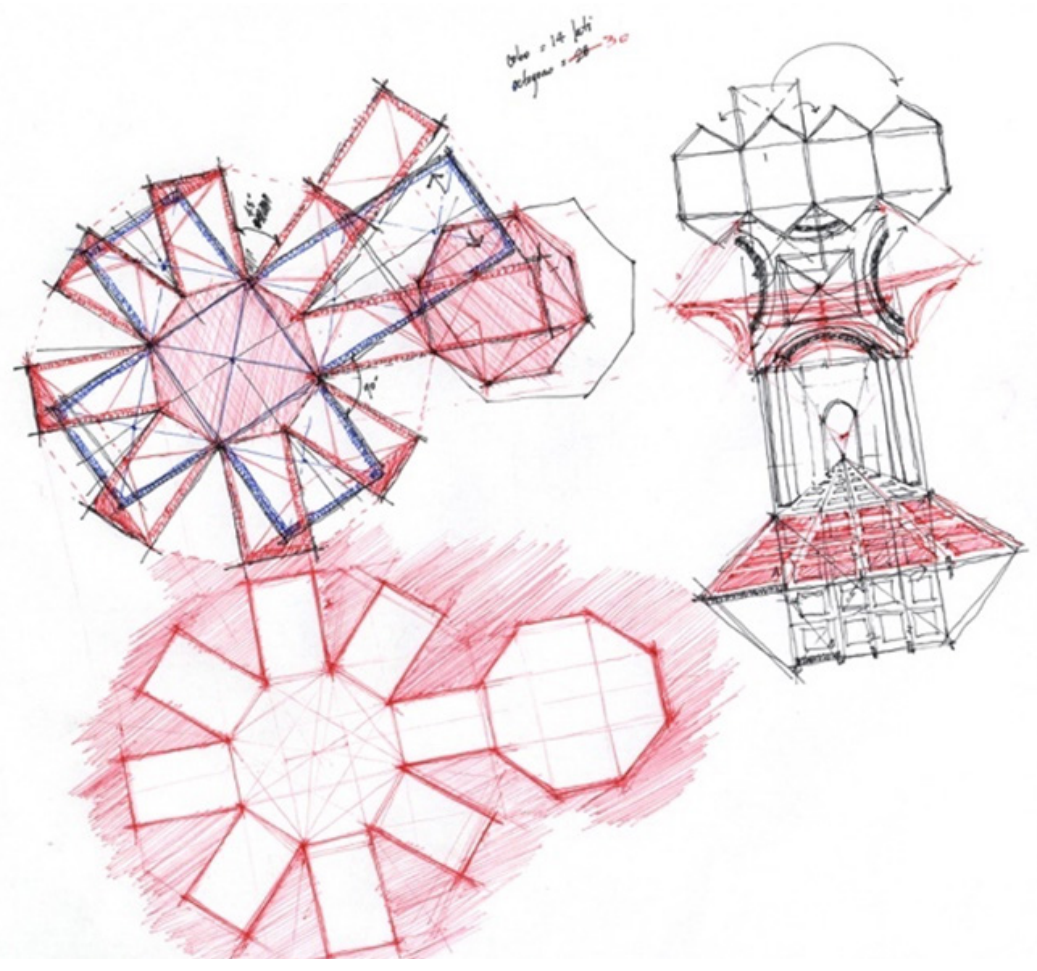

Figura 1: Pruebas con polígonos rebatidos, Laboratorio Modelli, UNISA (2017)

Fuente: Producción de Lucas Fabián Olivero

- Proyección Equirectangular: es una de las proyecciones utilizadas para representar una esfera abierta en el plano.

Esta estructura mantiene todas las líneas verticales, que coinciden con los meridianos, rectas. Las líneas horizontales, en cambio, resultan curvas y no coinciden con los paralelos. Éstos últimos, a su vez si resultan horizontales y dividen al plano en cuadrados iguales en la intersección con las primeras verticales. Puede verificarse fácilmente que esta proyección presenta como característica una gran deformación en las zonas de los polos y las formas allí contenidas, las cuales no tienen una fácil interpretación y lectura. La parte central es, a su vez, más cercana a una proyección cilíndrica. Si bien es más "entendible", no cobra tampoco entera dimensión si no es convirtiendo las líneas curvas a rectas y reduciendo el cono de visión. Para la reconstrucción de la anamorfosis se usa un programa de visualización esférica.

- Proyección Cúbica: se utiliza para representar un cubo abierto en el plano.

Se compone de un diagrama en cruz compuesto de las seis caras del cubo. Por ser una proyección plana, se pueden realizar entonces seis dibujos con los métodos tradicionales de la perspectiva como base. Si bien no sufre de grandes deformaciones, posee otras complicaciones como una mayor cantidad de discontinuidades, las cuales vendrán a complicar el proceso de montaje posterior. Una vez realizado el contenido, nuestra unidad de referencia será de todas maneras la equirectangular, por ser, entre otras ventajas, el input estándar para los motores de realidad virtual (Araújo, 2018a).

Para crear la inmersión y recrear el ambiente de forma interactiva, estas proyecciones serán modificadas informáticamente para mostrar solamente el "cono normal de visión" (flattening). Haciendo uso de visores de realidad virtual el dibujo puede incluso cobrar dimensión real a escala 1:1. Será, por lo tanto esta posterior visualización en la computadora lo que le da sentido al dibujo, ya que a pesar de estar hecho en papel y con técnicas analógicas, no tiene una lectura directa.

Serán presentadas a continuación dos experiencias que plasmarán los conceptos de base, sea con la proyección equirectangular como con la cúbica, que abrieron las puertas a la experimentación metodológica. Para salud de la ciencia, cabe aclarar que cuanto es aqui expuesto es el resultado de métodos de prueba y error, reportando el primer escalón, los primeros pasos que guiaron la descubierta de los fundamentos teóricos que sustenta esta nueva forma híbrida. Para una lectura más desarrollada metodológicamente se recomiendan los trabajos Drawing Equirectangular VR Panoramas (Araújo, 2018b) y CubeMe (Rossi, Barba y Olivero, 2018). 


\section{De las caras del cubo al arte $360^{1}$}

\subsection{Ver diferente}

A finales del 2015, surge para el Arq. Ing. Lucas Fabián Olivero la posibilidad de colaborar con el seguimiento de las tesis de grado de las estudiantes Carla Donato y Lucía Fernández, a realizarse en la Università degli Studi di Salerno (UNISA), Italia, en el marco del acuerdo de Doble Titulación que ésta mantiene con la Universidad Nacional de Córdoba, Argentina. La temática refería en particular la tecnología inmersiva, y una de las ideas que surge en ese momento es la aplicación de la gráfica analógica sobre una foto panorámica siguiendo la proyección equirectangular con el objetivo de usarlo para visualizar un proyecto arquitectónico en contexto.

Poco después y gracias a conocer a través de las redes sociales los primeros dibujos 360 del Arq. Bruno Sucurado, se extiende desde la UNISA una invitación para invitarlo a participar en la tesis de Donato. Con este gesto, se busca pensar en la evolución del dibujo tradicional a partir de estos claros indicios de una posible hibridación. Con esa óptica será planteado el curso de "Rilievo dell'Architettura", donde el módulo "Disegno dal Vero", dictado por Olivero y Sucurado, se pensó como un programa de trabajo conjunto para llevar a los estudiantes desde las primeras prácticas de "ablandamiento" de la mano hasta el dibujo esférico, pasando por perspectivas con múltiples puntos de fuga. Una serie de charlas preparatorias del curso condujo a evidenciar que las bases para pasar al 360 estaban sentadas, solamente era necesario un salto de visión, una interpretación diferente de la misma realidad.

Efectivamente, Olivero había ya intentado capturar especialmente en los dibujos de viaje por Europa desde 2011 (Barba, Ferraris y Olivero, 2014), hasta tres puntos de fuga. Se puede ver en estas piezas gráficas el uso (y muchas veces la exageración) del punto de fuga vertical, que le sirvieron para plasmar, extendiendo el normal plano de dibujo, las sensaciones abrumadoras de la arquitectura monumental.

Por otra parte, había juntado experiencia desde 2014 en la elaboración de visitas virtuales, adquisición de fotografías equirectangulares, así como también en el uso de instrumentales tales como Gigapan y Nodal Ninja. Esta práctica lo lleva finalmente a evidenciar que durante el proceso de stitching (elaboración de la fotografía panorámica solapando fotografías normales), existe también la posibilidad de exportar en los seis lados de un cubo. A partir del cubo se puede, por medio del procesamiento con software de stitching, obtener la equirectangular también sintetizando una relación biunívoca entre una y otra proyección.

${ }^{1}$ Experiencia del Arq. Ing. Lucas Fabián Olivero.
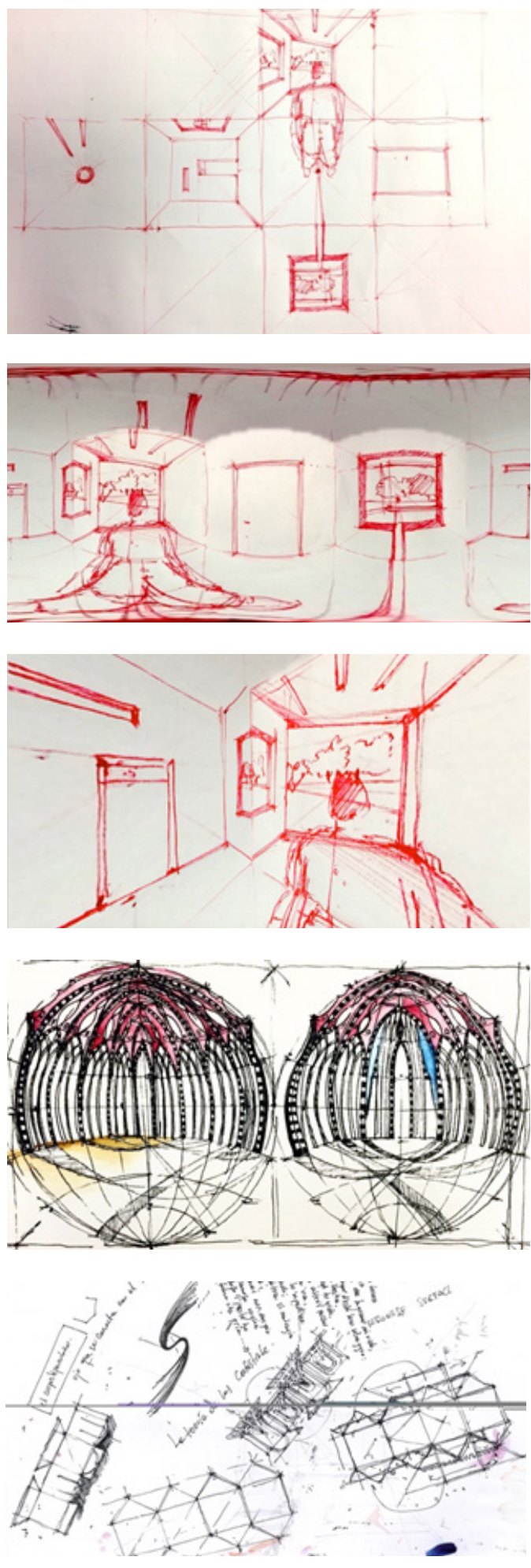

Figuras 2, 3, 4, 5 y 6: Primer cubo; Montaje de la equirectangular; Navegación con PTGUI (Windows); Pruebas a semiesferas; Pruebas con cubos desglosados aplicado en el ejemplo de figura 12, UNISA (2017). Fuente: Producción de Lucas Fabián Olivero. 
El cubo en informática, normalmente se utilizaba ya para la creación de los primeros ambientes virtuales en video juegos, concepto conocido como skybox. Durante la renderización del juego, el usuario es infinitamente pequeño (o el cubo infinitamente grande), por lo que al desplazarse "dentro" del cubo (lo que equivale a cambiar el punto de vista), no nota el fallo de la anamorfosis.

A partir de allí, una de las más tempranas revelaciones que surge para entender el dibujo usando el cubo es que, estando dentro de una habitación de base rectangular o cuadrada, "simplemente" deben dibujarse los seis puntos de fugas que nos rodean, uno por cada cara.

Del resultado de la experimentación gráfica con en el cubo, los polos (tan conflictivos con la proyección equirectangular), pueden dibujarse de forma más práctica siguiendo la metodología de una proyección plana. La perspectiva cónica conocida por todos es aplicable al cubo con ciertas salvedades que, si bien conllevan su complejidad, constituyen una base más intuitiva y progresiva para introducir los conceptos esféricos (Rossi et al, 2018).

Olivero realizó también algunas pequeñas pruebas de dibujos en semi-esferas siguiendo la proyección de azimut equidistante (lentes fisheye), así como también técnicas basadas en otras figuras ideales de polígonos. Estas pruebas por el momento no han seguido adelante, ya que encuentran su principal limitación en los bordes de discontinuidad. Sin embargo, la invitación a la Comunidad Científica Lectora es precisamente al estudio de posibles variaciones que puedan adaptarse, por ejemplo, a diferentes tipos de espacios. Salvedad primordial es que siendo la esfera el caso límite de cualquier superficie, toda otra superficie puede ser resumida en una esfera.

\subsection{El arte 360, rupturas y desafíos a la historia}

Completamente ya inserto en ambas metodologías, Olivero transfiere el dibujo 360 al campo del arte, donde recrea verdaderos espacios virtuales con atmósferas surreales. Puede verse cómo la expresión evoluciona desde las formas más rígidas (pegadas a la proyección) hacia expresiones más plásticas y espontáneas, una vez "dominada" la técnica. Con el proyecto LuFORIA, experimentará y producirá ricas producciones artísticas en paralelo con espectáculos de música en vivo.

Encontramos aquí otra utilidad y aplicación de estas producciones: videoclips también a 360 grados, que pueden navegarse en modalidad VR desde YouTube. Algunos artistas de vanguardia están explorando la técnica, como Caparezza o Muse que han hecho uso de cámaras a 360o, o Gorillaz que ha recreado y animado ambientes en forma digital. Tal es también el caso de cuanto ha producido junto con la artista emergente Elizabete Balčus (Olivero, Balčus, 2017). El método en este caso consistió en trabajar pivotando entre ambas proyecciones para producir los frames usando 360Toolkit, PTGui y Hugin. El montaje y la edición del video se realizan en formato equirectangular.
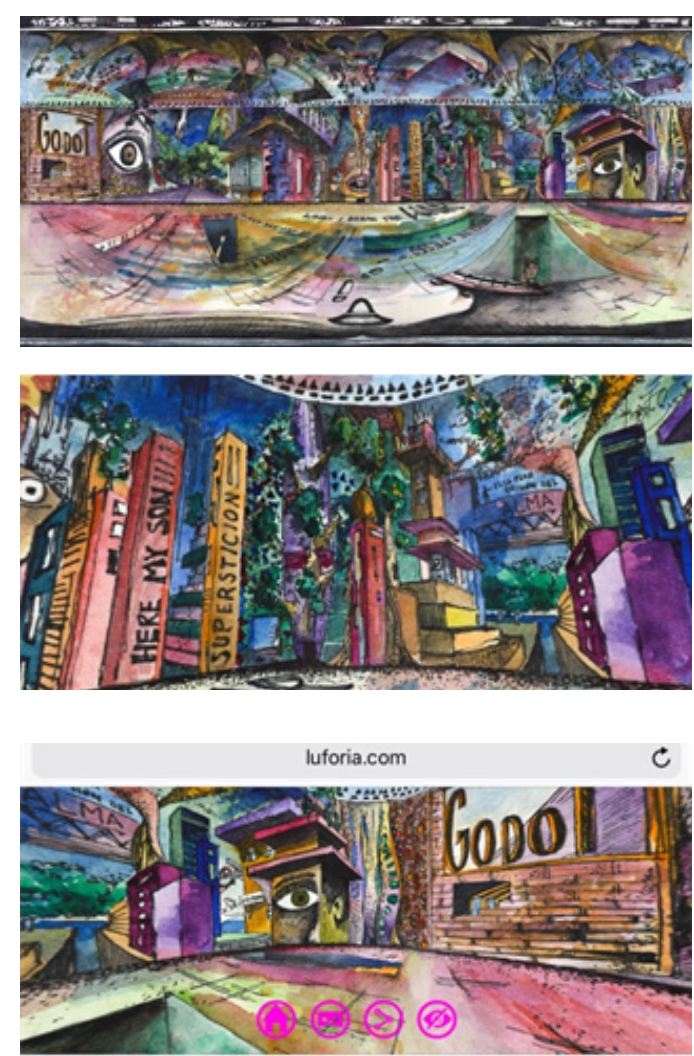

1) $m$
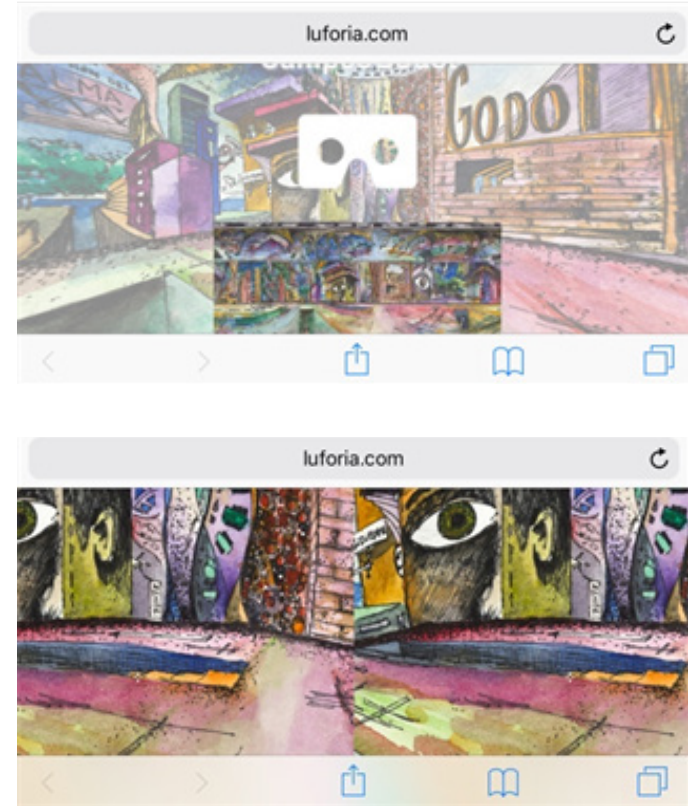

Figuras 7, 8, 9, 10 y 11: "Camposgodot", la primera expresión de arte analógica a 360 ; Navegación usando Panotour Pro; Navegación desde dispositivo móvil con iOS; Selección de la modalidad VR; Visión estereoscópica VR, Salerno (2017)

Fuente: Producción de Lucas Fabián Olivero 
Realizar estas obras directamente en proyección equirectangular es lo que las convierte en una de las partes más críticas de este desarrollo. Si recapitulamos en la historia podemos ver el gran salto que la sociedad ha dado al asimilar, estudiar y codificar la estructura de la perspectiva. Con este gesto, nos ampliamos a un nuevo concepto de representación donde tenemos a mano una estructura que se nutre de los mismos principios pero que alcanza una potencia mucho mayor.

Podemos entonces con esto lograr otro salto, dando uso a todos los años de experiencias y prácticas de las artes plásticas hasta hoy desarrollados. No bastará, sin embargo, que la grilla de una proyección sea la guía del artista: es menester que el conocimiento de su composición se haga intuición, para evitar caer en la ceguera del mero talento pictórico.

\subsection{El dibujo en las seis caras del cubo, algunos detalles de la técnica}

Como preanunciado, la práctica consiste en dibujar los seis lados de forma individual, en un cuadrado perfecto cada uno y con ciertas salvedades. Se aclara que la técnica misma está en fase de experimentación y desarrollo en literatura (Rossi et al., 2018), por lo que este intento de definición de una metodología no es exhaustivo completamente y es más bien una primera aproximación conceptual. La ocasión es útil, sin embargo, para sentar las primeras bases de referencia.

La línea de horizonte resulta la intersección de un plano que pasa por el centro volumétrico del cubo en las caras horizontales, por lo tanto, será una línea horizontal que pasa siempre por el baricentro de cada una de las cuatro caras horizontales.

Esto puede ser un poco engañoso en algunos casos, por ejemplo, en un dibujo tradicional si uno quisiera hacer más "dramática" la escena, tendería a bajar la línea de horizonte e inclinar las verticales. Este gesto, natural como recurso gráfico, no se corresponde con la estructura 360 y deriva en errores de visualización ya que la anamorfosis que la sustenta se define informáticamente siempre en el centro volumétrico.

Por otro lado, la proyección de una línea que pasa hacia resulta que, si la línea es horizontal al observador en la cara frontal, su continuación en las caras de la izquierda y la derecha serán inclinadas e irán hacia el centro geométrico de dichas caras, coincidentes en este caso con los puntos de fuga.
Comprender este aspecto es entender que estamos dibujando escenas diferentes por cada lado del cubo, y asimilar el "giro" necesario para capturar lo que nos rodea. Las figuras 12, 13 y 14 ilustran algunos bosquejos en los cuales, por clara falta de conocimiento de la estructura, no se tuvieron en cuenta estos factores.

Toda línea que toca un borde de una de las caras y no termina allí, debe tener una continuación en la cara adyacente. Siguiendo este principio se deberá entonces tener el cuidado de continuar todas las líneas dibujadas que tocarán un borde, de lo contrario se resaltará la lectura de una discontinuidad no pensada.
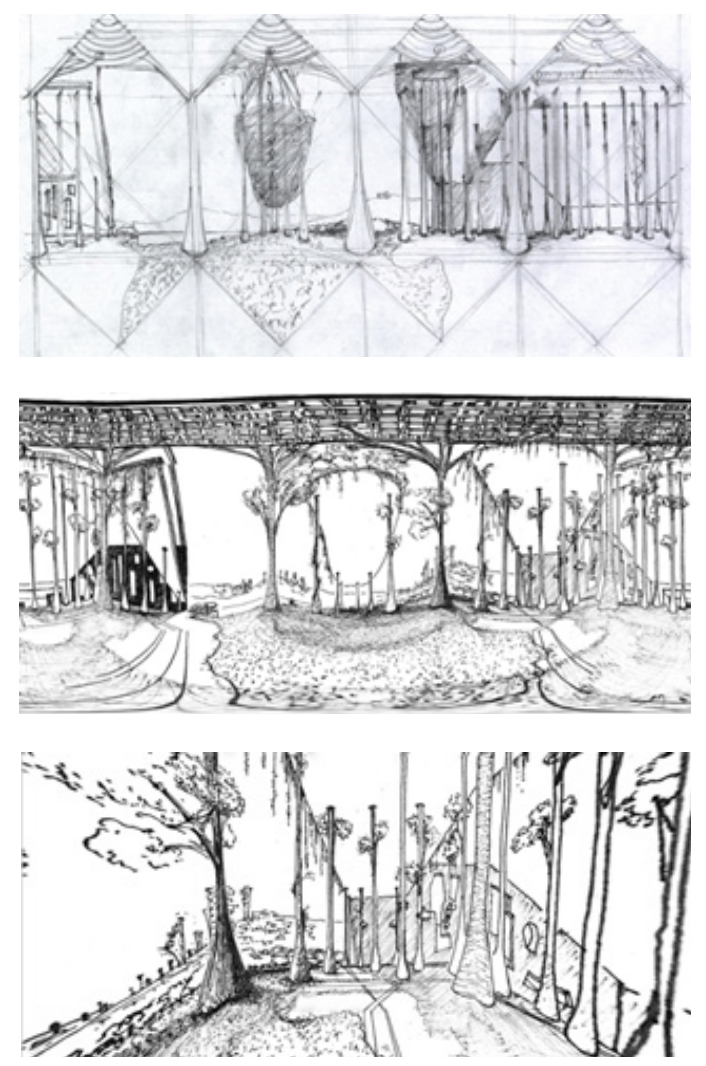

Figura 12, 13 y 14: "Minerva" (nótese el error en la línea de horizonte no alineada con el centro del cubo); Montaje de la equirectangular; Navegación desde la computadora donde se evidencia el error de la elaboración, Laboratorio Modelli, UNISA (2017).

Fuente: Producción de Lucas Fabián Olivero. 


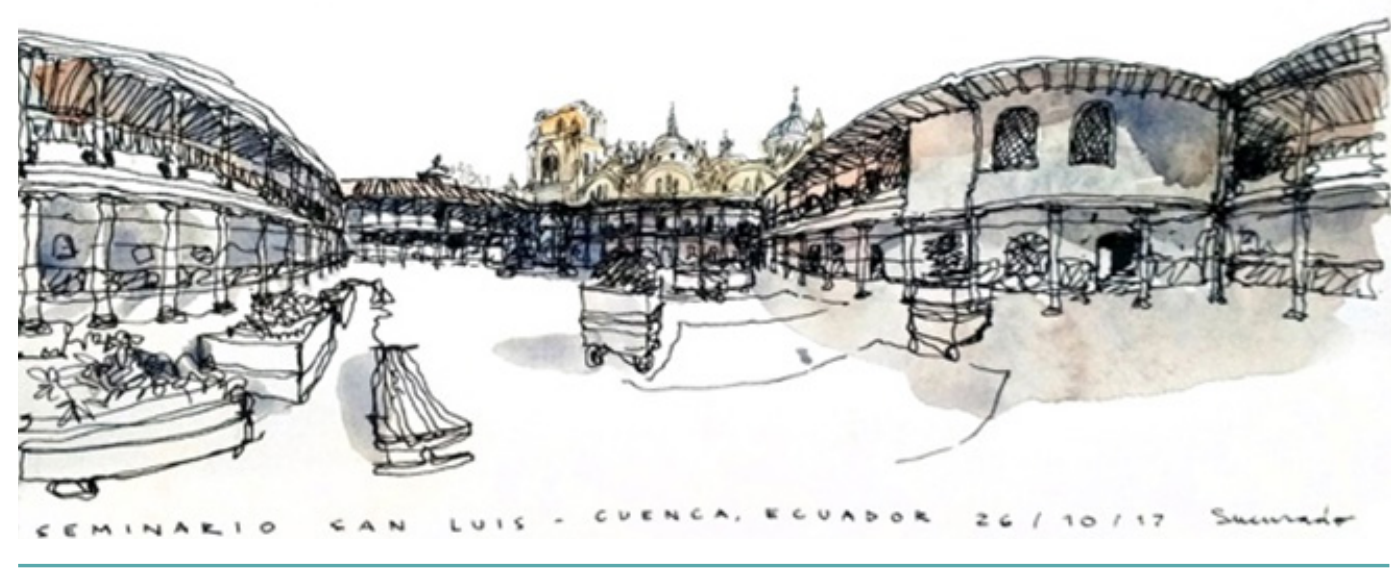

Figura 15: Seminario San Luis, Cuenca, Ecuador (2017).

Fuente: Producción de Bruno Sucurado.
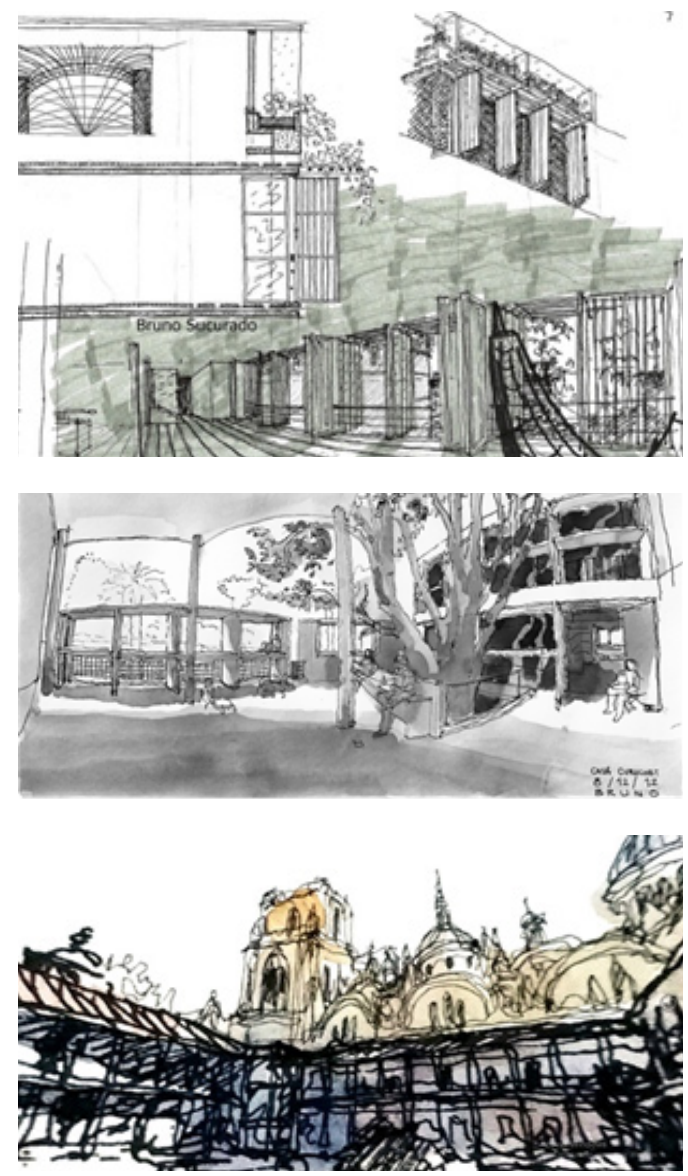

Figuras 16, 17 y 18: Procesos de búsqueda, Buenos Aires; Dibujos in situ de la Casa Curutchet, La Plata (2008 2015); Navegación con Roundme de la figura 15. Fuente: Producción de Bruno Sucurado.

Experiencia del Arq. Bruno Sucurado
4. De la perspectiva

curvilínea al panorama esférico $^{2}$

Bruno Sucurado se vio, siendo estudiante de arquitectura en ciudad de La Plata, Argentina, frente a la inquietud de poder lograr una perspectiva de 360 en un único dibujo. Así fue que la perspectiva curvilínea le marcó un camino para expandir el campo visual y lograr incluso una imagen más consistente con la realidad observada a pesar de las deformaciones que provoca la curvatura.

Un incremento de estas aplicaciones tuvo lugar en los últimos años de su carrera, mostrando escenas panorámicas difícilmente representables con fugas rectas, barriendo generalmente no más de 180 o en horizontal. En este proceso de búsqueda, vislumbró el concepto de una esfera como contenedora de todo el campo visual, atravesada por un eje cartesiano XYZ que determina en su intersección con dicha esfera seis puntos de fuga óptimos de un espacio ideal triortogonal.

Estudiando luego la fotografía panorámica llega a conocer la proyección equirectangular que la cartografía determina como una de las múltiples formas de desplegar la superficie de una esfera (como la tierra) en un plano. Esta proyección le fue de crucial utilidad para plasmar, en un solo plano, toda la realidad observada alrededor del observador.

Lo crucial era entonces dibujar las deformaciones curvas con cierta precisión.

Con esta pauta en mente surgen las primeras pruebas para representar el entero campo visual a 360o. Es interesante notar que, gracias a la distorsión excesiva en el dibujo, la mente ha de forzarse a hacer pensando en la futura visualización digital. El espacio real como tal, es casi irreconocible directamente cuando se lo ve en proyección equirectangular. 

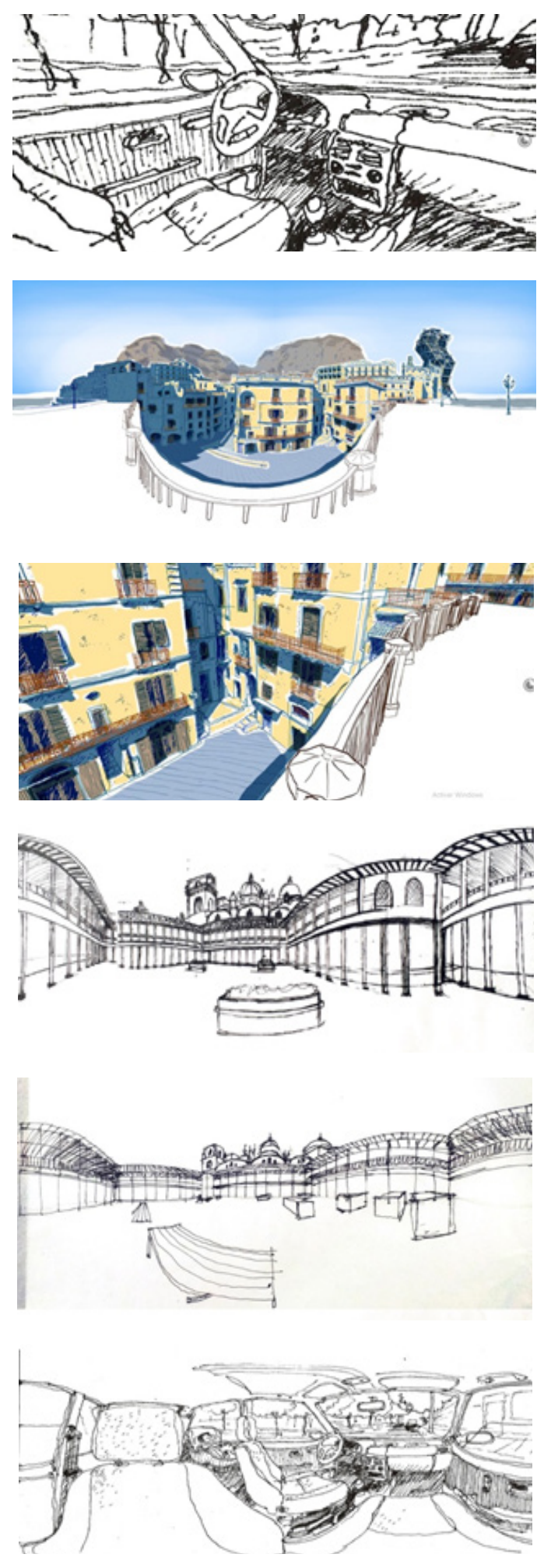

Figuras 19, 20, 21, 22, 23 y 24: Primer 360; Navegación con Roundme Buenos Aires. (2016); 360 de Atrani, Italia, para el proyecto "Gigakahn"; Navegación web; 360 de los estudiantes del seminario en Cuenca.

Fuente: Bruno Sucurado, Luis Fernández Parra y otros.
El paso final fue montar el dibujo plano en una esfera digital y poder navegarlo informáticamente.

A través de la mencionada invitación hecha por la UNISA (sección 3.1), Sucurado participa de eventos coordinados por el profesor Salvatore Barba, entre los cuales realiza algunos dibujos 360 de la Costiera Amalfitana que fueron posteriormente incluidos en el virtual tour "Gigakahn" resultante de la tesis (Donato, 2017) y el curso de "Rilievo dell'Architettura", donde el arquitecto expuso estas primeras experiencias en las aulas italianas.

Otra invitación llegará entonces a Sucurado, quien presenta sus talleres en Ecuador en el Congreso Internacional de Comunicación Gráfica Arquitectónica. Allí confirmará lo que en Salerno ya había notado: los alumnos de Cuenca descubren que la técnica no requiere virtuosismo ni excesivos conocimientos técnicos más de los que ya tenían a su disposición. Efectivamente logran captar sin grandes inconvenientes espacios a 360 grados dentro de la Facultad de Arquitectura y del Convento de San Luis, en el corazón de la ciudad.

\section{Aplicaciones prácticas}

\subsection{Experiencias didácticas}

En 2016 se desarrolla el ya nombrado curso en la Universidad de Salerno para estudiantes del quinto año de Ingeniería edilicia-arquitectónica. Se dicta en forma conjunta por Sucurado y Olivero, quienes articulan un programa con una parte introductoria de dibujo a mano alzada (con prácticas desde uno hasta seis puntos de fuga) para presentar, como corolario, el 360 en ambas técnicas.

Los resultados fueron sorprendentes incluso para los mismos estudiantes, quienes en un principio se mostraron un poco escépticos y pasmados por pensar en dibujar todo lo que a su alrededor veían. Poco a poco, con la práctica fueron aprehendiendo la grilla equirectangular y percibiendo su relación con el espacio representado. A medida que adquirían confianza iban agregando expresividad y estilos particulares.

El curso estaba compuesto por estudiantes de las carreras de ingeniería y de arquitectura, manifestando estos últimos una mayor facilidad para entender los conceptos y desarrollar técnicas gráficas, consecuencia directa de una formación más fuerte en el dibujo a mano alzada. Esto, sin embargo, no frenó ni inhibió a los "menos entrenados" que osaron ir más allá de sus propios límites con óptimos resultados.

Otra experiencia fue una presentación en la Univrsidad degli Studi della Campania "Luigi Vanvitelli" (UNICAMPANIA) hecha por Olivero, en el marco de las presentaciones Ilamadas "I Giovedi del Dottorato". Se presentó, a muy grandes rasgos, la historia de cómo se llega al 360 con una aplicación práctica de dibujo en el 
momento. Seminarios y cursos han sido impartidos desde entonces: México (Sucurado), en diferentes universidades italianas (Olivero) y en sede de congresos internacionales (UID Milano, Olivero, 2018).

Observaciones tempranas sobre la experiencia en el aula restituyen que, a paridad de tamaño de hoja, los alumnos tienden a dibujar los objetos más grandes con el cubo ya que resulta difícil tener consciencia de que un cuadrado representa un plano de dibujo correspondiente a las proyecciones en ángulo de visión de $90^{\circ}$.
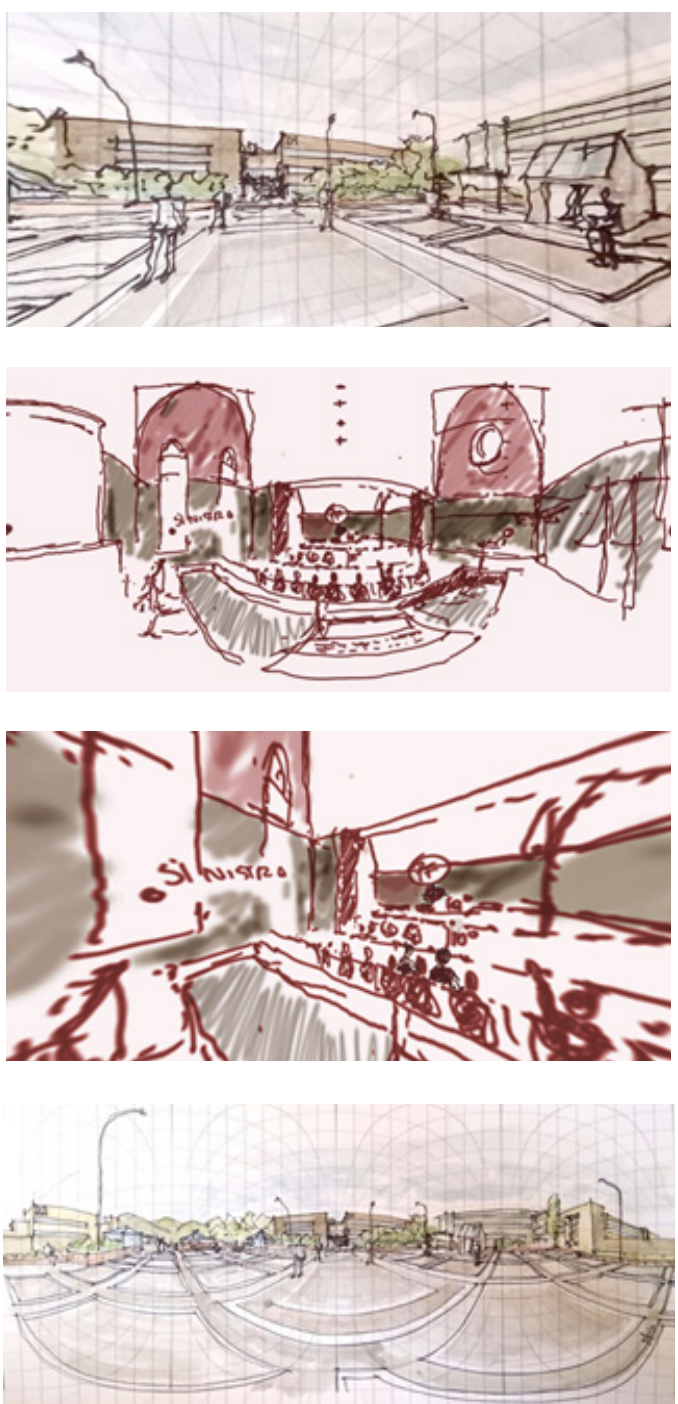

Figuras 25, 26, 27 y 28: Piazza dei Sapere, Campus de la UNISA; Navegación con Roundme; Aula Magna UNICAMPANIA; Navegación con PTGUI, Aversa (2017) Fuente: Agostina Ciancaglini y Lucas Fabián Olivero.
Existe también una tendencia del aprendiz a confundir el contenedor (cubo) con el contenido: cuando se dibuja una habitación rectangular, por ejemplo, se confunden los bordes de las caras del cubo con las aristas de la habitación.

\subsection{Levantamientos arquitectónicos}

El sintetizar el espacio real dibujándolo, constituye una forma de apreciación personal y única, la elaboración de un único modelo conceptual (Arnheim, 1954), por lo que el valor de dicha realización estimula la creatividad y la crítica de los elementos que se representan.
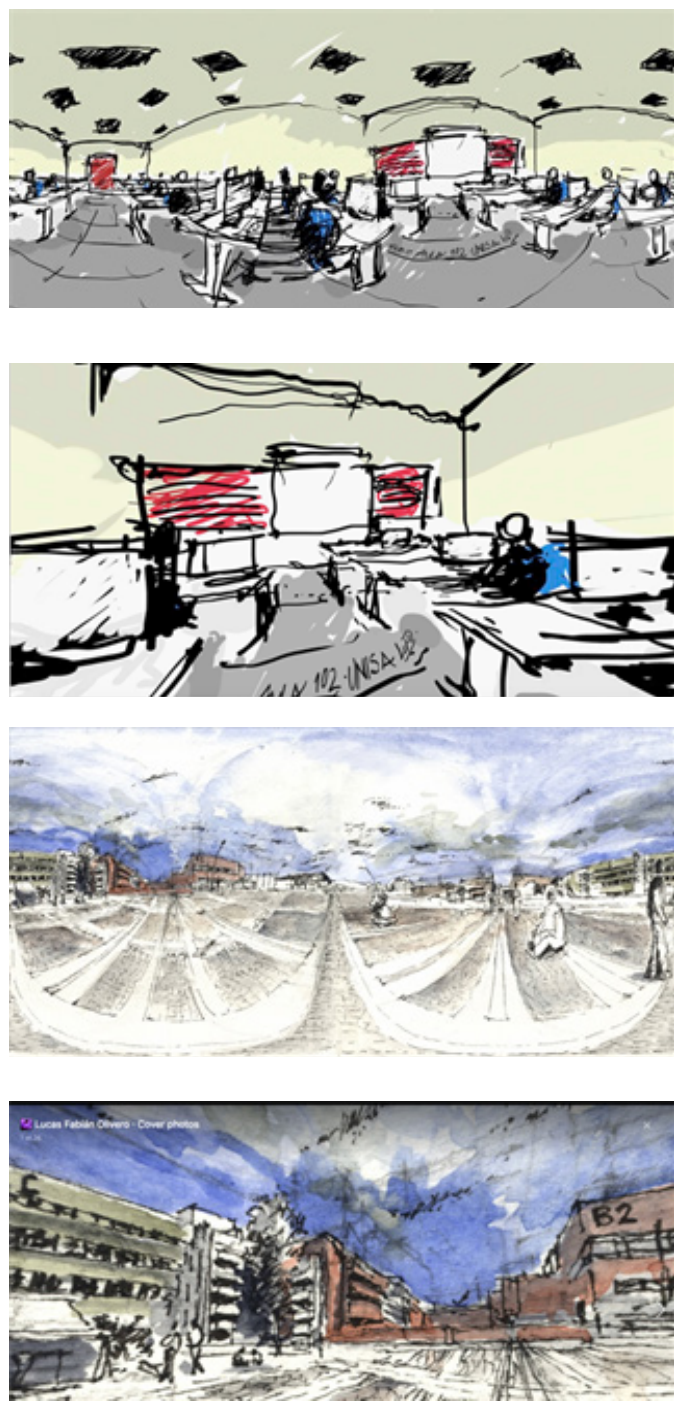

Figuras 29, 30, 31 y 32: Aula 102; Navegación con uso de FSP Viewer (MAC); Piazza dei Sapere; Navegación usando Facebook, Campus de la UNISA (2017).

Fuente: Producción de Lucas Fabián Olivero 
Como connotación práctica, en los diferentes momentos en los cuales han sido dictados los cursos para enseñar estas técnicas, el dibujo de los espacios reales se convierte también en la forma más simple para que la persona solo se concentre en aprender la metodología y la estructura de fondo, relacionando la realidad directa con el dibujo que se produce.

\subsection{Pensar espacialidades: la arquitectura de la mente}

La gráfica analógica asociada al proceso de pensar la arquitectura representa, aún hoy, una de las mejores herramientas para capturar la sinapsis entre proceso creativo y visualización. Es simple constatar el hecho de que cuando un cliente se presenta en el estudio en busca de un proyecto, son los bocetos los primeros en ser testigos de este diálogo entre proyectista y cliente. Mal que le pese al mundo digital, quizás siga siendo todavía la mejor forma de hacerlo por la simple razón de que e diálogo es espontáneo, la comunicación es instantánea y difícilmente el cliente tenga paciencia para esperar la modelación 3D y la elaboración de planos.

Proponemos, entonces, el estudio de los dibujos 360 aumentando el abanico de posibilidades expresivas, pero sobre todo veloces, que el arquitecto puede utilizar. En este caso, la visualización sería semi-espontánea (vista la necesidad de tener que al menos recortar la foto para cargarla y navegarla). Se gana en crear con una misma pieza gráfica la inmersión en la obra.

\subsection{Arte}

Hemos ya hecho una introducción acerca de algunas experiencias relacionadas con el arte en la sección 3.2 agregaremos la importancia que la tecnología inmersiva está teniendo en ámbitos internacionales combinadas con las AR (Augmented Reality), VR (Virtual Reality) y MR (Mixed Reality). Se están creando a partir de aquí notables proyectos, tales como The Armory Show en New York o de valorización museal como II Bello o il Vero en Italia.

Otros, en cambio, como The Starry Night de Magic Shanghai usan esta tecnología y las animaciones computarizadas para recrear, por ejemplo, los universos capturados por Van Gogh. Se reafirma entonces, también en este campo, la necesidad de una exploración profunda de las técnicas híbridas que permita la participación e inserción dentro de las nuevas modalidades para vivir el arte.

Se destaca en este inciso que existen ciertos plugins (como Oniride) basados en Photoshop que trabajan directamente en la creación sobre el cubo. Otros, como Sketch 360, trabaja directamente sobre la grilla equirectangular. En ambos casos, se trabaja sobre una base raster, marcándose en ambos casos una carencia de soportes vectoriales.
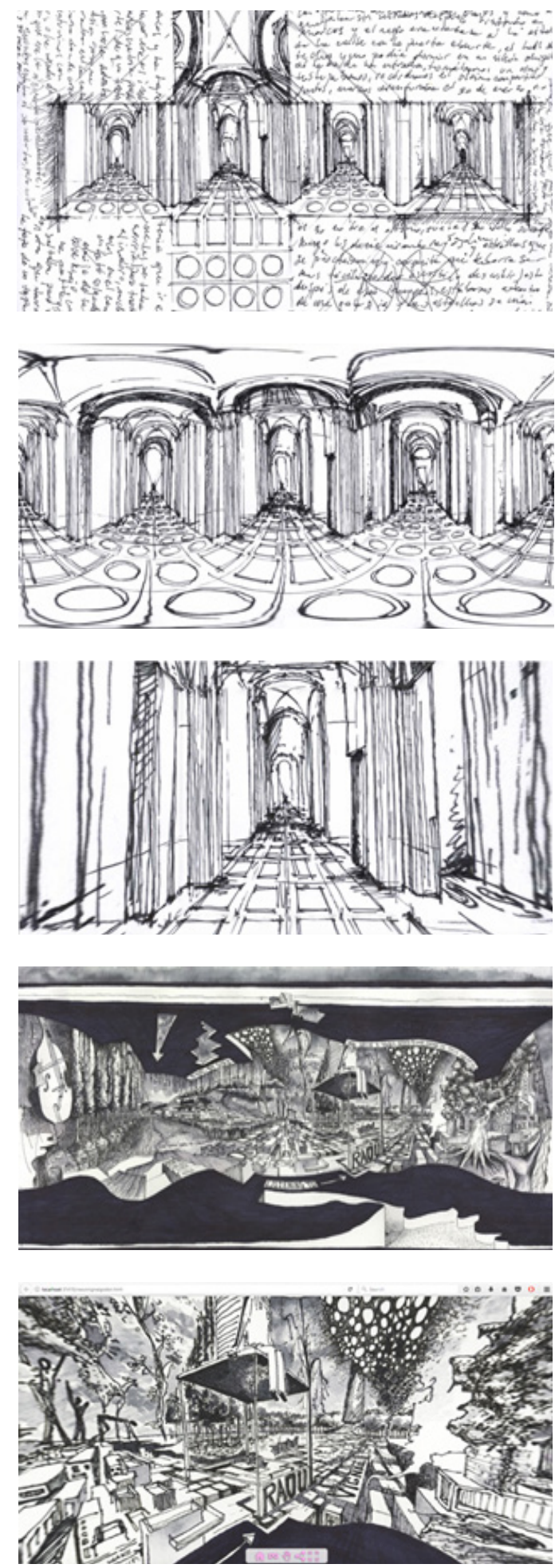

Figuras 33, 34, 35, 36 y 37: "Requiem", exploración espacial; Montaje de la equirectangular; Navegación con uso de FSP Viewer (MAC); "Waking Up 360 Times", Navegación usando Panotour Pro, Italia (2017).

Fuente: Producción de Lucas Fabián Olivero. 


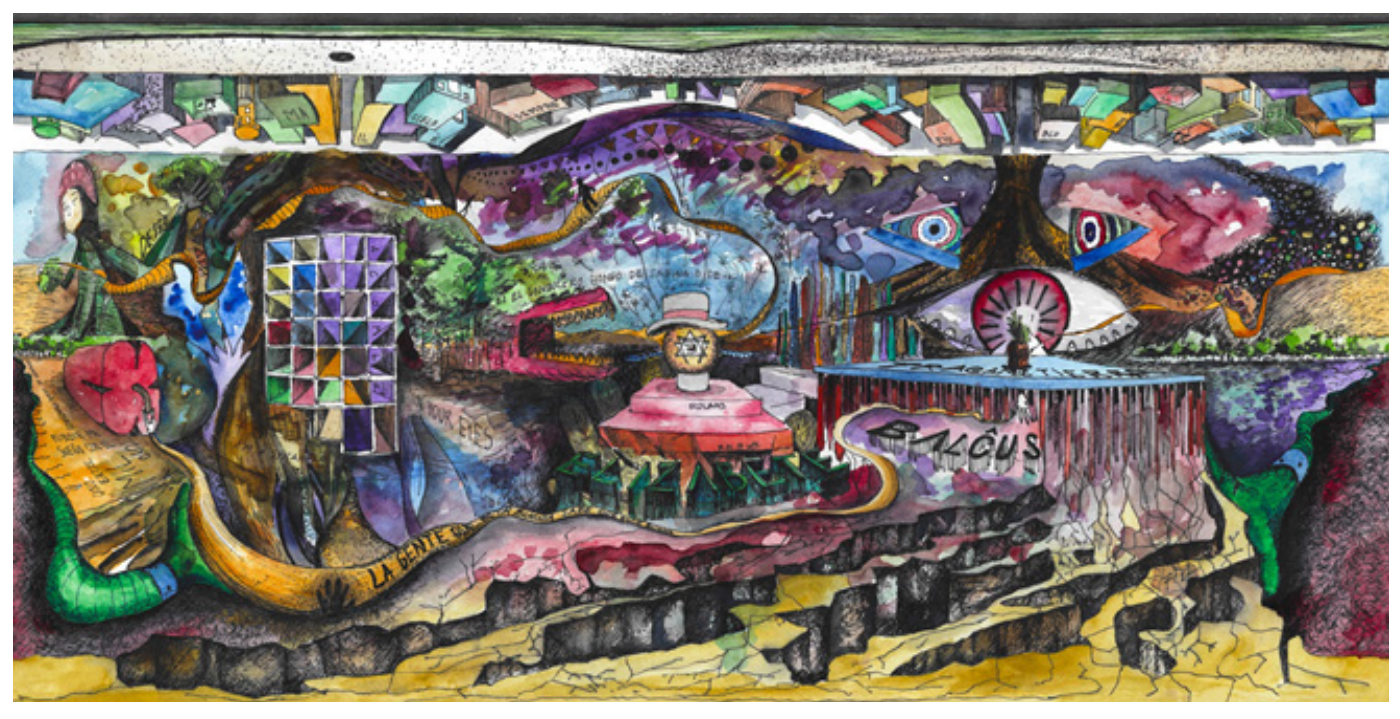

Figura 38: "Elizabete: Hat, Fruit or Flute" arte equirectangular analógica, acuarelas y tinta, Salerno, Italia (2017) Fuente: Elaboración de Lucas Fabián Olivero

\section{6. ¿Proyección cúbica o equirectangular?}

Llegados a este punto se presenta una comparación prematura de ambas técnicas con el objeto de resaltar algunas características favorables y complicaciones de cada una de ellas. Algunos de los factores que pueden afectar a la hora de la elección pueden ser:

La precisión técnica: por cuanto lo desarrollado en la literatura hasta el momento, la perspectiva en proyección equirectangular posee más desarrollo analítico matemático (Araújo, 2018a). Sin embargo, la deformación en torno a los polos resulta complicada de controlar sin esa base de precisión (grilla de curvas geodésicas) y la visualización durante la navegación digital se ve afectada en estas zonas, ya que la deformación del píxel es muy grande. La equirectangular, para poder realizar un bosquejo más rápido y preciso al mismo tiempo se ve limitada al grado de conocimiento del desarrollo de la curvatura. Por otra parte, esta proyección contiene la mínima expresión de discontinuidades: una sola arista dividida en dos laterales y los dos puntos, superior e inferior, estirados y abiertos, que deberán luego cerrarse en sí mismos.

La perspectiva en proyección cúbica, por su parte, una vez que vea resuelto su lado analítico matemático y geométrico descriptivo (de copioso desarrollo, por cierto), podrá capturar precisión técnica sin grandes alteraciones en la visualización (la deformación del píxel es casi nula). El cubo conlleva, sin embargo, un mayor grado de atención y de trabajo por las discontinuidades que presenta ya que a la hora de cerrarse presenta siete bordes de discontinuidad. La ilusión de la continuidad (diferencia entre contenido y contenedor) dependerá de la precisión con las que las líneas se continúen.

El tiempo de ejecución: si el destino del dibujo es presentar una idea rápida, esbozar un proyecto en pocas líneas, entonces la equirectangular (con las salvedades de su precisión) quizás sea más adecuada, entre otras cosas porque es más simple navegarla de forma directa (FSP Viewer, 360Toolkit, PTViewer). El cubo, por su parte, con excepción de la herramienta 360Toolkit, requiere un recorte previo y la creación de la panorámica. Estas consideraciones son importantes ya que afectan el tiempo, por ejemplo, a la hora de visualizar una pequeña modificación (para un mayor detalle sobre el montaje del cubo puede consultarse CubeME de Rossi et al., 2018).

Los medios disponibles: la equirectangular aprovecha mejor el papel, basta definir un contenedor $2 \times 1$ (con poco desperdicio en una hoja A4); el cubo, por su parte, resulta más complejo de diagramar en el soporte.

El confort para adaptarse al 360: quienes posean una práctica fluida de perspectiva y logren dibujar curvas con docilidad, seguramente no experimentarán mayores complicaciones al pasar a la proyección equirectangular. Quienes, en cambio se encuentren en dificultad, deberían quizás trabajar con el cubo hasta que comiencen a adaptarse poco a poco a la anamorfosis de la esfera. 


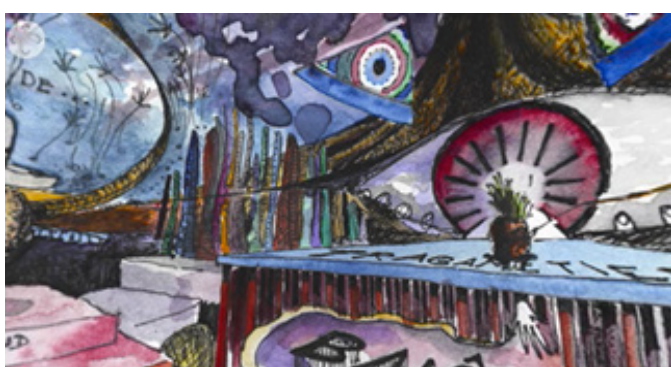

Figura 39: Navegación del video "They're Coming" para Elizabete Balčus usando YouTube (2017).

Fuente: Producción de Lucas Fabián Olivero.

\section{Primeras conclusiones}

Llegados a este punto, nos preguntamos: ¿Por qué deberíamos seguir insistiendo en una forma de expresión analógica habiendo descubierto la versatilidad de los modelos informáticos puros?

UNO: En la representación, luego de llegar a realizar este tipo de producciones habiendo alterado los métodos convencionales de dibujo, vemos que los dibujos a 360 grados pueden aportar una nueva forma de producción híbrida cuya comprensión, intuitiva "a simple ojo" y con las herramientas de decodificación que nuestro cerebro tiene hoy, no es posible si no se poseen los conocimientos de anamorfosis. En el vaivén inherente a la complejidad entre el analógico y el digital, nuestro cerebro comenzará a decodificar y entender una nueva dimensión y una metodología para representar la misma realidad, pero evolucionada al siglo XXI y asociada a lo digital. Lejos entonces de negar la contemporaneidad, se sirve de las nuevas tecnologías, se abraza a ellas para enriquecerse y abrir nuevas puertas.

DOS: En la práctica gráfica se ha escrito mucho acerca del dibujo a mano alzada; sabemos y conocemos muchas de sus bondades y la potencia creativa que éste aporta a quien lo desarrolla y lo practica, siendo recurso de estimulación artística pero también de almacenamiento de información en manera veloz e intuitiva. Esta práctica complementa a los procesos racionales rígidos aportando libertad de expresión y riqueza en las posibilidades de experimentación artística, material, arquitectónica y espacial. No es banal entonces destacar que el dibujo a 360 grados representa un uso ampliado de lo autógrafo, evitando la pérdida del estímulo creativo por la decadencia de uso.

TRES: La ingeniería en background, sea de la perspectiva equirectangular como de la cúbica, abre profundos e importantes campos de estudio de la disciplina gráfica que serán seguramente tenidos en cuenta para posteriores desarrollos y experiencias académicas.

CUATRO: En la arquitectura, habiendo explorado el uso de las fotografías panorámicas, aun siendo un dato de calidad fotográfica, rara vez se usa para el proyecto arquitectónico. Sentadas las bases científicas, el dibujo sobre esas fotos serviría como forma de aprovechar ese material testimonio de la realidad material.

CINCO: En el arte, el dibujo 360 presenta una nueva ruta para recorrer, abriendo oportunidades inéditas como por ejemplo los videos 360 o las creaciones de ambientes. La utilización de la inmersión para la creación artística nos conecta al momento del Renacimiento, cuando el conocimiento de la perspectiva y su técnica gestaban los grandes artistas. Los movimientos liberatorios $y$ desestructurados del siglo XX se basaron, en cambio, en las rupturas, hoy ambas cosas se tocan y fusionan generando un nuevo movimiento artístico.

\section{Agradecimientos}

A la profesora Adriana Rossi de la Università della Campania "Luigi Vanvitelli", al profesor António Bandeira Araújo de la Universidade Aberta de Lisboa (ambos tutores de Lucas Fabián Olivero en su tesis doctoral) y al profesor Salvatore Barba del Laboratorio Modelli del Dipartimento di Ingegneria Civile de la Università degli Studi di Salerno, por el apoyo brindado, por las oportunidades en las aulas y la apertura multidisciplinar, tan importante para el avance académico y científico en nuestros tiempos.

\section{Referencias bibliográficas}

\section{Libros y Artículos}

- Araújo, A. B. (2018a). Drawing Equirectangular VR Panoramas with Ruler, Compass, and Protractor. Journal of Science and Technology of the Arts, 10(1), 15-27. doi: 10.7559/citarj.v10i1.471

- Araújo, A. B. (2018b). Ruler, compass, and nail: constructing a total spherical perspective. Journal of Mathematics and the Arts, 12(2-3), 144-169. doi: 10.1080/17513472.2018.1469378

- Arnheim, A. (1954). Art and Visual Perception. A psychology of the creative eye. (2a ed.). California, Estados Unidos: University of California Press.

- Barba S. (2014). Tecniche di image editing: un possibile "work flow" per le AP. Roma, Italia: Sapienza Università Editrice. doi: 10.13133/978-88-98533-45-9

- Barba, S., Ferraris, R. y Olivero, L. F. (2014). La bitácora de viaje: aprehensión y transferencia en los procesos de aprendizaje. En Á. Melián García (Ed.), El dibujo de viaje de los arquitectos. Gran Canaria, España: Servicio de Publicaciones y Difusión Científica de la Universidad de Las Palmas de Gran Canaria. 
- Barre, A., Flocon, A., y Bouligand, G. (1967). La Perspective curviligne: de l'espace visuel à l'image construite. Paris, Francia: Flammarion.

- Cabezos Bernal, P., Cisneros Vivó, J., y Soler Sanz, F. (2014). Anamorfosis, su historia y evolución. EGA Expresión Gráfica Arquitectónica, (23). doi: 10.4995/ ega.2014.2184

- Cabezos Bernal, P. y Cisneros Vivó J. (2015). Stereoscopy in Descriptive Geometry books. EGA Expresión Gráfica Arquitectónica, (26) 242-255. doi: 10.4995/ ega.2015.4058

- Cardone, V. (1999). Modelli grafici e modelli informatici. Proiezione e immagine, la logica della rappresentazione. Actas del Seminario Didáctico. Nápoles, Italia: Arte Tipografica.

- Della Francesca, P. (1474 aprox.). De prospectiva pingendi (ed. Chiara Gizzi 2016). Venezia, Italia: Università Ca' Foscari.

- Greene, N. (1986). Environment Mapping and Other Applications of World Projections. IEEE Computer Graphics and Applications, 6(11), 21-29. doi: 10.1109/ MCG.1986.276658

- Israel, J.H., Wiese, E., Mateescu, M., Zöllner, C., y Stark, R. (2009). Investigating three-dimensional sketching for early conceptual design - Results from expert discussions and user studies, Computers \& Graphics, 33, (4), 462-473. doi: 10.1016/j.cag.2009.05.005.

- Masetti, M. (2014). La prospettiva e la costruzione dello spazio figurativo. Italia: Youcanprint.

- Muñoz Viñas, S. (2004). Teoría Contemporánea de la restauración. Madrid, España: Síntesis S.A.

- Pozzo, A. (1693). Perspectiva pictorum et architectorum. Roma, Italia: Typis Joannis Jacobi Komarek.

- Rossi A. (2017). Immersive high resolution photographs for cultural heritage. Drawing / Disegno books series. V. 2. Italia: libreriauniversitaria.it

- Rossi, A., Barba, S. y Olivero, L. F. (2018). "CubeME", a variation for an immaterial rebuilding. En Rappresentazione / Materiale / Immateriale. Drawing as (in)tangible representation (pp. 31-36). Roma, Italia: Gangemi Editore International.
- Rossi, D. (2018). Brand new: panorama. L'immagine continua per il disegno della realta virtuale / Brand new: panorama. Continuous image to shape virtual reality. En Rappresentazione / Materiale / Immateriale. Drawing as (in)tangible representation (pp. 1389-1396). Roma, Italia: Gangemi Editore International.

- Sandnes. F.E. (2016). PanoramaGrid: A Graph Paper Tracing Framework for Sketching 360-degree Immersed Experiences. En Proceedings of the International Working Conference on Advanced Visual Interfaces (pp. 342-343). Bari, Italy: ACM. doi: 10.1145/2909132.2926058.

\section{Trabajos de tesis}

- Olivero, L.F. (2015). II rilievo architettonico: le problematiche infografiche, teoriche e pratiche, della fruizione del dato acquisito. Università degli Studi di Salerno, Universidad Nacional de Córdoba.

- Donato, C. (2017). Gigakahn, un progetto grafico di documentazione, comunicazione e valorizzazione ispirato al viaggio di Louis Kahn in Costiera Amalfitana. Università degli Studi di Salerno, Universidad Nacional de Córdoba.

\section{Sitios web}

- Wikipedia. (2018). Cube Mapping. https://en.wikipedia. org/w/index. php?title=Cube mapping\&oldid=837243861

- Wikipedia. (2018). Skybox (Video Games). https:// en. wikipedia.org/w/index. php?title=Skybox_(video_ games)\&oldid=825433763.

- Olivero L.F., Balčus E. (2018). They're Coming, 360 Handmade Artwork \& Music Video. goo.gl/DxxfkF

- Van Gogh- The Starry Night VR- 3D 360o experience https:/ goo.gl/BS66xK

- Visualizador gratuito de imágenes panorámicas equirectangulares sin metadatos https://roundme.com

Proyecto LuFORIA https://www.luforia.com

Simon Sinek \& The Millennial Question https://goo.gl/X1yxYb

Fundación, Gala-Salvador Dalí. "Dalí. Estereoscopías. La pintura en tres dimensiones." Dali exhibitions. Recuperado de https://exhibitions.salvador-dali.org/es/daliestereoscopies/.

Get Sketch 360. (n.d.). Recuperado de https://www microsoft.com/en-us/p/sketch-360/9p89s 2qlh11 